B. ELLMAN ET AL.: DIAGNOSTIC PNEUMOPERITONEUM IN AMOEBIC LIVER ABSCESS

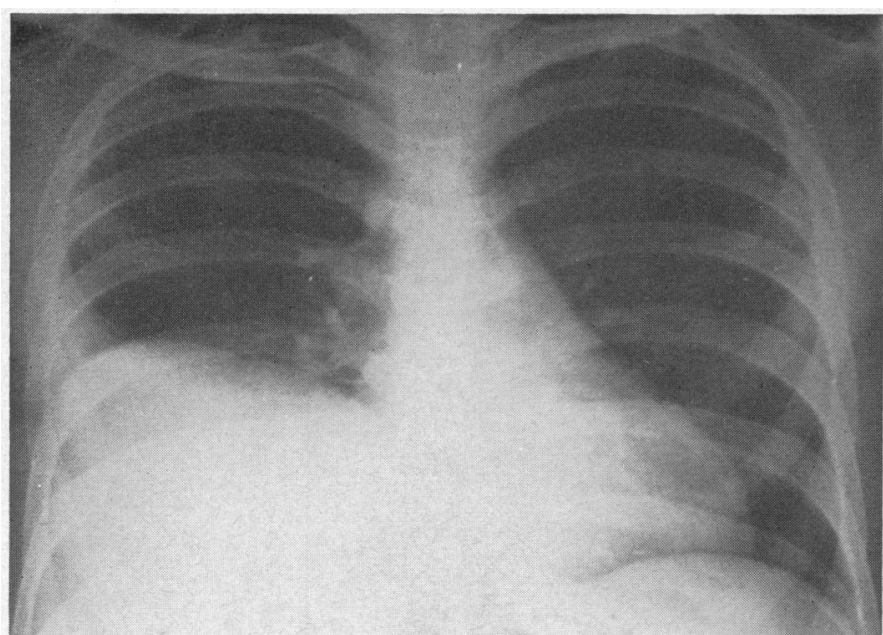

FIG. $1 a$

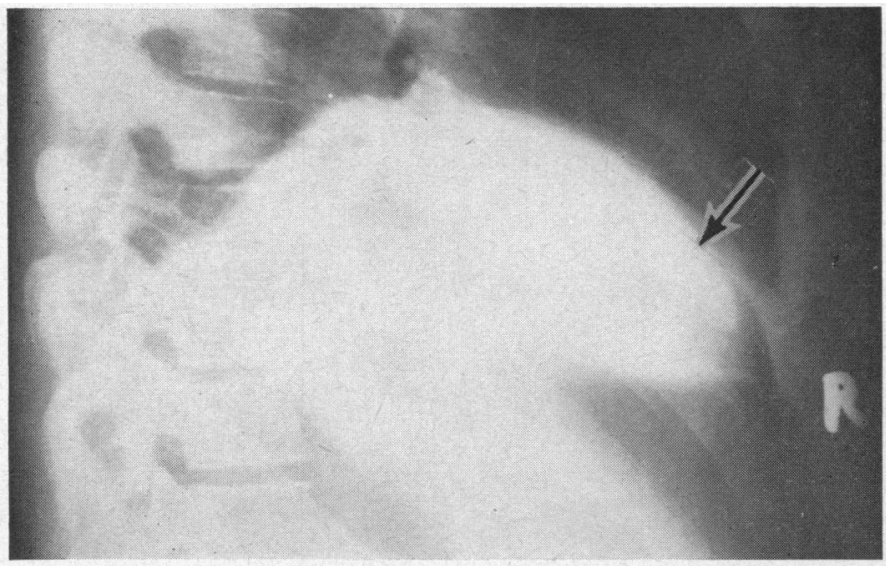

Fig. $1 c$

FIG. 2.-Case 2. Right basal pneumonia. (a) Postero-anterior radiograph showing elevation of the right hemidiaphragm with irregular consolidation in the adjacent lung. (b) and (c) Antero-posterior and right lateral erect radiographs after diagnostic pneumoperitoneum, showing absence of adhesions between liver and diaphragm.

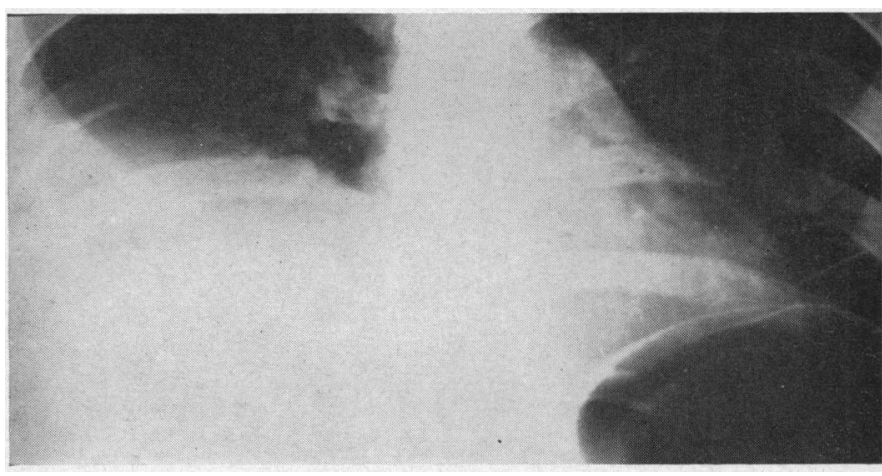

FIG. $1 b$

FIG. 1.-Case 1. Proved amoebic liver abscess. (a) Postero-anterior radiograph showing elevation of the right hemidiaphragm with small lamella effusion. (b) and (c) Antero-posterior and right lateral erect radiographs after diagnostic pneumoperitoneum, showing adhesions between liver and diaphragm.

FIG. $2 c$

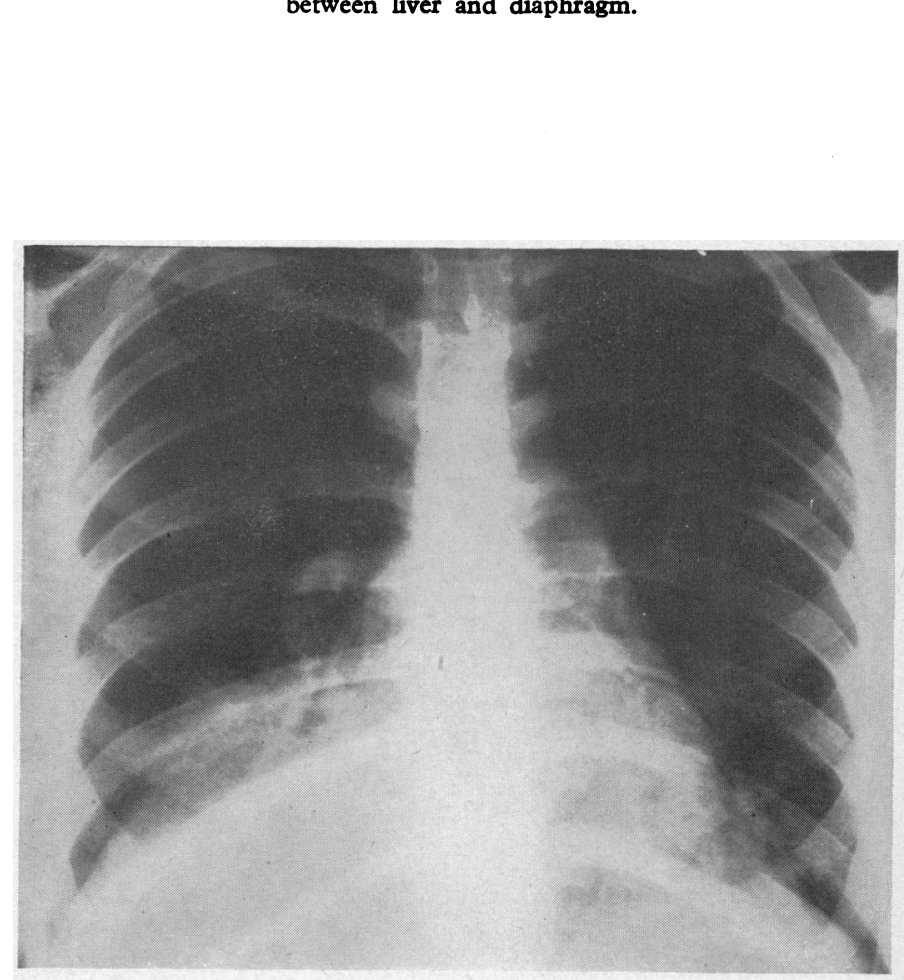

FIG. $2 a$

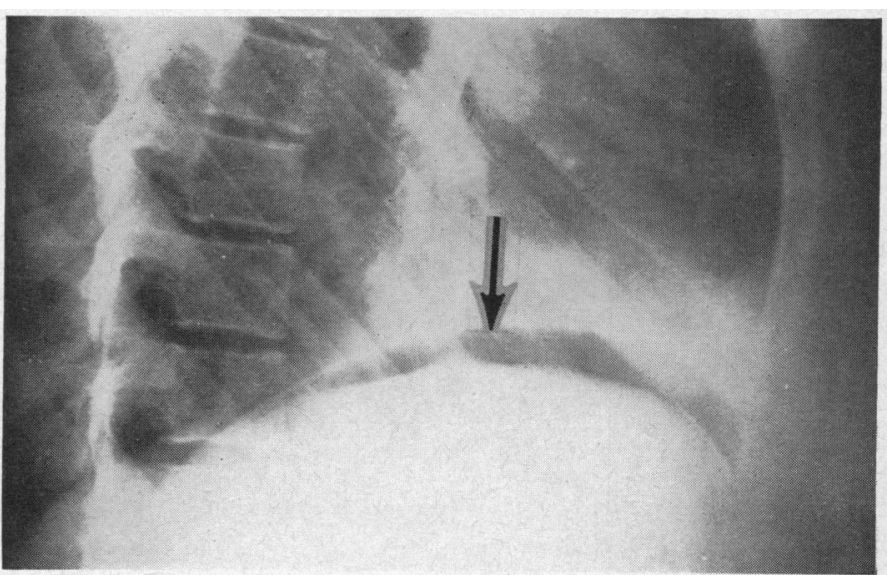

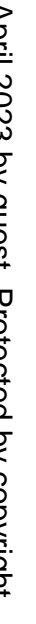

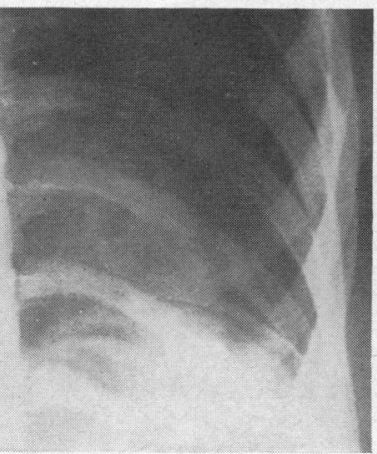


Cooper, L. Z., Green, R. N., Krugman, S., Giles, J. P., and Mirick, G. S. (1965). Morbidity and Mortality Weekly Report, Communicable Disease Center, U.S. Public Health Service, 14, 44.

Deutsch, H. F., and Morton, J. I. (1957). Science, 125, 600.

Dudgeon, J. A., Butler, N. R., and Plotkin, S. A. (1964). Brit. med. 7., 2, 155 .

Gregg, N. M. (1941). Trans. ophthal. Soc. Aust., 3, 35.

Ingalls, T. H., Babbott, F. L., Hampson, K. W., and Gordon, J. E. (1960). Amer. F. med. Sci., 239, 363.

Korones, S., Ainger, L., and Roane, J. (1965). 75th Annual Meeting of the American Pediatric Society, Philadelphia, Pa. (Abstracts), p. 39. May, 1965.

Lündström, R. (1962). Acta paediat. (Uppsala), 51, Suppl. No. 133.

Manson, M. M., Logan, W. P. D., and Loy, R. M. (1960). Reports on Public Health and Medical Subiects, No. 101. H.M.S.O., London. Morbidity and Mortality Weekly Report (1964). Communicable Disease Center, U.S. Public Health Service, 13, 93.

Parkman, P. D., Buescher, E. L., and Artenstein, M. S. (1962). Proc. Soc. exp. Biol. (N.Y.), 111, 225 .

Phillips, C. A., Behbehani, A. M., Johnson, L. W., and Melnick, J. L. (1965). F.' Amer. med. Ass., i91, 615.

Plotkin, S. A. (1964). Ibid., 190, 265.

(1965). Unpublished data.

Dudgeon, J. A., and Ramsay, A. M. (1963). Brit. med. F., 2, 1296.

\author{
Oski, F. A., Hartnett, E. M., Hervada, A. R., Friedman, S., and \\ Gowing, J. (1965). 7. Pediat., 67, 182. \\ Rorke, L. (1965). Unpublished data. \\ Rubella Symposium (1965). 35th Annual Meeting of the Society for \\ Pediatric Research and the 75th Annual Meeting of the American \\ Pediatric Society, Philadelphia, Pa. (Abstracts), May, 1965. \\ Rudolph, A. J., Yow, M. D., Phillips, C. A., Desmond, M. M., Blattner, \\ R. J., and Melnick, J. L. (1965). F. Amer. med. Ass., 191, 843. \\ Schiff, G. M., Sutherland, J. M., Light, I. J., and Bloom, J. C. (1965). \\ 35th Annual Meeting of the Society for Pediatric Research, Phila- \\ delphia, Pa. (Abstracts), p. 47. May, 1965. \\ delphia, Pa. (Abstracts), p. 47.
Selzer, G. (1963). Lancet, 2, 336. \\ Sever, J. L. (1965). Personal communication. \\ Huebner, R. H., Castellano, G. A., Sarma, P. S., Fabiyi, A., Schiff, \\ G. M., and Cusumano, C. L. (1965). Science, 148, 385 . \\ Schiff, G. M., Bell, J. A., and Huebner, R. J. (1964). F. Pediat., \\ 65, 1027 (Abst.). \\ Sheridan, M. D. (1964). Brit. med. F., 2, 536. \\ Weller, T. H., Alford, C. A., and Neva, F. A. (1964). New Engl. 7. \\ Med., 270, 1039. \\ - and Neva, F. A. (1962). Proc. Soc. exp. Biol. (N.Y.), 111, 215. \\ Zinkham, W. H., and Medearis, D. N. (1965). 75th Annual Meeting of \\ the American Pediatric Society, Philadelphia, Pa. (Abstracts), p. 2. \\ May, 1965.
}

\title{
Diagnostic Pneumoperitoneum in Amoebic Liver Abscess*
}

\author{
B. ELLMAN, $\dagger$ M.B., D.M.R.D. ; I. N. MCLEOD, $\ddagger$ M.D., F.C.P.(S.A.); S. J. POWELL,§ M.D., M.R.C.P.ED.
}

[With Special Plate]

Brit. med. F., 1965, 2, 1406-1407

In those regions where liver abscess is common the differentiation of a subphrenic source from primary pulmonary disease in the right lower zone of the chest is a frequent problem in diagnosis. The clinical features may be equivocal, and radiology is necessary to locate the precise anatomical site of such lesions. In Durban, where amoebic liver abscess is common among Africans, we have found that the conventional radiological techniques of postero-anterior and lateral films and of screening for diaphragmatic movement may not assist in distinguishing supradiaphragmatic right basal lung conditions from extension of a liver abscess through the diaphragm into the thorax. In some cases it is impossible to visualize the diaphragm adequately, and in other instances screening for diaphragmatic movement and estimation of diaphragmatic level has been found unreliable. At necropsy we have noted that extension of amoebic liver abscess into the thorax results in adhesions forming between the superior surface of the liver and the diaphragm. However, when an inflammatory process originates in the lung there is little tendency for downward extension to occur through the diaphragm and the liver remains free of adhesions. By delineating the superior surface of the liver and demonstrating the presence or absence of adhesions to the diaphragm, pneumoperitoneum, with carbon dioxide as contrast medium, offered a radiological method of potential diagnostic value. This paper reports our experience of such study.

Though the technique of pneumoperitoneal air insufflation was first described by Kelling (1902) and introduced to diagnostic radiology in 1910 , there has been some reluctance to employ it in diagnosis, possibly because of misapprehension regarding the complications. Initially air or oxygen was

* Requests for reprints of this paper should be addressed to Dr. S. J. Powell, Amoebiasis Research Unit, P.O. Box 1035, Durban, South Africa.

† Department of Radiology, University of Natal Medical School, Durban, South Africa.

¥Department of Medicine, University of Natal Medical School, Durban,

South Africa.
Department of Medicine, University of Natal Medical School, and Amoebiasis Research Unit, P.O. Box 1035, Durban, South Africa.

The Amoebiasis Research Unit is sponsored by the following bodies: the South African Council for Scientific and Industrial Research, the Natal Provincial Administration, the University of Natal, and the United States Public Health Service (Grant A1, 01592). employed as the gas medium, but in 1921 Alvarez introduced the use of carbon dioxide, thereby eliminating one of the major hazards of the procedure-namely, fatal air embolism. Stein (1951), reviewing the literature, concluded that the procedure was free from serious pleural and pulmonary complications.

\section{Material}

Thirty patients were selected in whom difficulty was being experienced in establishing a possible subphrenic cause of their disease. As the object of pneumoperitoneum was not to replace but to supplement the simpler techniques, only those patients were studied in whom the clinical findings, teleradiographs, and screening of the chest were equivocal or unhelpful in diagnosis. On clinical examination the majority presented with symptoms and signs of variable duration referable to the right lower chest and right upper quadrant of the abdomen. In some the abdominal signs were dominant, in others findings in the right lower chest predominated.

The primary object of pneumoperitoneum in each instance was to distinguish between supradiaphragmatic extension of an amoebic liver abscess and primary pulmonary or pleural disease by success or failure in demonstrating adherence between the superior surface of the liver and the diaphragm. Secondary objectives were to determine the position and contour of the diaphragm and to delineate any abnormalities in hepatic contour.

\section{Method}

Preliminary chest screening was carried out and control postero-anterior and lateral chest radiographs were taken. No pre-operative preparation other than sedation was required.

As many of the complications of pneumoperitoneum are related to faulty placement of the needle the abdomen was carefully palpated to ensure that no mass was present at the puncture site. Except when a mass or scar was present the site of election was 1 in. $(2.5 \mathrm{~cm}$.) to the left and 1 in. above or below the umbilicus. Under local anaesthesia the peritoneum was punctured by means of a short bevelled needle, and, to 
ensure that a vessel or viscus had not been entered, aspiration was attempted prior to injection of the gas. Correct placement of the needle was confirmed by auscultating the abdomen, when a characteristic sibilant sound could be heard during the injection. A simplified apparatus for the injection of gas was used and from 250 to $1,500 \mathrm{ml}$. of carbon dioxide was introduced. The amount used depended on the patient's tolerance, the injection being discontinued as soon as discomfort was felt. In view of the rapid absorption of the gas medium the procedure was done on the radiographic table. Positioning depended on the suspected site of the lesion, the patient lying prone, supine, or with the right side elevated. Radiographs were taken with either horizontal or vertical beams and further radiographs done with the patient antero-posterior or slightly oblique in the erect position. The most useful views were achieved in the erect position or with the tube horizontal.

\section{Results}

In 17 of the 30 patients adhesions were demonstrated between the superior surface of the liver and the diaphragm (see Special Plate, Fig. $1 \mathrm{a}-\mathrm{c})$. All except one of these 17 patients responded well to specific anti-amoebic therapy (emetine hydrochloride and chloroquine), and in eight the subsequent aspiration of typical amoebic pus in the vicinity of the adhesions provided proof of the diagnosis of amoebic liver abscess. Eight were shown to have generalized or localized elevation of the right dome of the diaphragm and five had a localized convex deformity of the superior surface of the liver. In two of the latter tomography demonstrated cavities which subsequently yielded amoebic pus on aspiration in the areas of the deformity. The remaining patient, who did not respond to amoebicides, had undergone a subtotal gastrectomy for a perforated gastric ulcer four months previously, and, after response to antibiotic therapy, the diagnosis of pyogenic subphrenic abscess was made.

In the 13 patients without demonstrable adhesions no confirmatory evidence of amoebiasis was obtained and the final diagnosis consisted of the following entities: pneumonia with or without effusion, tuberculous pleural effusion and empyema, carcinoma of the lung, and right basal bronchiectasis (see Special Plate, Fig. 2 a-c).

\section{Discussion}

The use of diagnostic pneumoperitoneum has been comprehensively reviewed by Maxfield and McIlwain (1944) and by Lumsden and Truelove (1957). The commonly listed complications are all rare (Stein, 1951) with the exception of transitory subphrenic or shoulder-tip pain, which was experienced by two patients in our series. The injection of air or oxygen into a viscus or vessel may produce air embolism, and Burman (1956) noted this occurrence in six instances, two of which were fatal, in a review of 53,543 refills and 426 pneumoperitoneum inductions with the use of air, but the employment of carbon dioxide almost completely eliminates this risk (Lumsden and Truelove, 1957), and we have found this technique to be safe and simple. Chatterjee, discussing the report of Tandon and Khanna (1960), has suggested that induction of pneumoperitoneum in patients with amoebic liver abscess might result in the breaking down of hepatodiaphragmatic adhesions and rupture of the liver abscess into the peritoneal cavity. In no instance was this possible complication encountered in our series, and, with the relatively small quantities of rapidly absorbed gas used, we feel its occurrence is highly unlikely.

Like ourselves, Clark et al. (1948) and Keeley (1955) were impressed with the potential of pneumoperitoneum as an aid in the diagnosis of subphrenic lesions, particularly amoebic liver abscess. Nevertheless, we would emphasize that it should be used in conjunction with, and not in place of, other investigations. The procedure has a definite place in the investigation of suspected liver abscess, but its use is necessary only where inadequate or equivocal information is provided by the clinical findings and simpler radiological techniques. In such instances pneumoperitoneum provides a relatively simple diagnostic aid which can be undertaken in ill patients with little discomfort, though it should be borne in mind that in liver abscess its value is largely limited to the demonstration of lesions on the superior surface of the liver, and, should a lesion be demonstrated, it does not differentiate the aetiology. Equivocal results may be encountered if insufficient carbon dioxide is introduced, and in our experience it is more difficult to demonstrate adhesions arising from the left lobe of the liver. However, the common site for amoebic liver abscess is the right lobe with extension upwards to the right dome of the diaphragm, and adhesions in this situation can usually be readily visualized. Newer techniques such as ultrasonic diagnosis (Wang et al., 1964) and isotope hepatoscanning (Marberg and Czerniak, 1964) are now being employed and will doubtless prove of value, but the simplicity and accuracy of pneumoperitoneum in selected cases commend themselves.

More than 500 patients with amoebic liver abscess are seen annually at this hospital. In the vast majority the clinical picture is characteristic. Proof of diagnosis in some is obtained by the aspiration of typical pus and in others by the anchovy character of the pus coughed through a hepatobronchial fistula. In most of the remainder the diaghosis is based on the clinical findings and response to amoebicidal treatment, though recently developed serological tests are proving of value (Maddison, 1965 ; Powell et al., 1965). The radiological changes at the lung base which occur in $75 \%$ of patients (Wilmot, 1962) and which have been well documented (Druckmann and Schorr, 1944; Munk, 1944 ; Isaac, 1945) are often of extreme importance in diagnosis. Nevertheless there remains a small group in which diagnosis is obscure and the distinction of lesions of subphrenic origin from primary thoracic conditions by pneumoperitoneum has proved of value in these patients.

\section{Summary}

In Durban, South Africa, where amoebic liver abscess is common, difficulty in differentiating primary pulmonary disease in the right lower zone of the chest from subphrenic lesions is not infrequent. Diagnostic pneumoperitoneum was done in 30 selected patients, and adhesions demonstrated between the superior surface of the liver and the diaphragm in 17 . The procedure was found to be safe, well tolerated, and of assistance in diagnosis.

We wish to thank Drs. S. C. Truelove and K. Lumsden, of the Radcliffe Infirmary, Oxford, for suggesting this study, and Professor W. Sacks and E. B. Adams, Heads of the Departments of Radiology and Medicine, University of Natal Medical School, Durban, for their interest. We are grateful for the co-operation of the medical staff for giving access to patients under their care, and to Dr. R Nupen, Acting Superintendent,. King Edward VIII Hospital, Durban, for providing facilities.

\section{REFERENCES}

Alvarez, W. C. (1921). Amer. 7. Roentgenol., 8, 71.

Burman, D. (1956). Thorax, 11, 49.

Clark, R. H. P., Bercovitz, Z. T., and Jones, R. F. (1948). Amer. F. trop. Med. Hyg., 28, 545 .

Druckmann, A., and Schorr, S. (1944). Harefuah, 26, 183.

Isaac, F. (1945). Radiology, 45, 581.

Keeley, K. J. (1955). Brit med. 7., 2, 1029.

Kelling, G. (1902). Münch. med. W Wchr., 49, 21.

Lumsden, K., and Truelove, S. C. (1957). Brit. ₹. Radiol., 30, 516

Maddison, S. E. (1965). Exp. Parasit., 16, 224.

Mardison, S. E. (1965). Exp. Parasit., 16, 224.

Marfield, J., R., iun., and McIlwain, A. J. (1944). Radiology, 42, 346.

Maxfield, J. R., jun., and McIlwain, A. J. (1944). Radiology, 42, 346.
Munk, J. (1944). Brit. J. Radiol., 17, 48.

Powell, S. J., Maddison, S. E., Wilmot, A. J., and Elsdon-Dew, R. (1965). Lancet, 2, 602 .

Stein, H. F. (1951). Amer. Rev. Tuberc., 64, 645.

Tandon, R. N., and Khanna, B. K. (1960). Ann. Biochem., 22, 415.

Wang, Hsin-fang, Wang, Cha-en, Chang, Ch'ing-r'ing, Kao, Ju-yüeh, Yü, Lu-min, and Chiang, Yung-nien (1964). Chin. med. Э., 83, 133.

Wilmot, A. J. (1962). Clinical Amoebiasis, p. 87. Blackwell, Oxford. 